\title{
Clinical Study on Treatment of 39 Cases of Uremic Restless Leg Syndrome by Rouxiujiang Decoction Combined with Acupoint Massage
}

\author{
Hongxia Xie ${ }^{1}$, Xin Pang ${ }^{1}$, Xiaoyong Chen ${ }^{1}$, Dongyang Li ${ }^{1}$, Jianwei Zhang ${ }^{1}$, Yan Zhang*, ${ }^{2}$ \\ ${ }^{1}$ Henan Province Hospital of Traditional Chinese Medicine, The Second Affiliated Hospital of Henan \\ University of Traditional Chinese Medicine, Zhengzhou, Henan, 450003
}

${ }^{2}$ The First Affiliated Hospital of Henan University of Traditional Chinese Medicine, Zhengzhou, Henan, 450000

Keywords: Rouxiujiang Decoction; Acupressure; Uremic Restless Leg Syndrome

\begin{abstract}
Objective: To explore the clinical effects of the treatment of uremic restless leg syndrome by the treatment of Rouxiujiang Decoction and acupoint massage. Methods: A total of 78 patients with uremic restless legs syndrome from April 2016 to January 2018 were enrolled in our hospital. The random number table method was divided into control group $(n=39)$ and study group $(n=39)$. Hemodialysis treatment was adopted in both groups. On the basis of this, the control group was treated with acupoint massage. The study group was treated with Rouxiujiang Decoction on the basis of the control group, and all were treated for 2 months. After the end of the treatment period, the clinical efficacy and the degree of illness (RLS) before and after treatment and the score of sleep quality (PSQI) were calculated. Results: The total effective rate of study group was 94.87\% (37/39) higher than that of control group $76.92 \%(30 / 39, \mathrm{P}<0.05)$. There was no significant difference between the two groups before treatment $(\mathrm{P}>0.05)$. The RLS scores of the group were lower than those before the treatment, and the study group was lower than the control group $(\mathrm{P}<0.05)$. There was no significant difference between the PSQI scores of the two groups before treatment $(\mathrm{P}>0.05)$. After the treatment, the PSQI scores of the two groups were better than before treatment. Decreased, and the study group was lower than the control group $(\mathrm{P}<0.05)$. Conclusion The combination of acupoint massage and Rouxiujiang Decoction urgent soup treatment of uremic restless legs syndrome can effectively relieve the clinical symptoms of patients, reduce the degree of illness, improve the therapeutic effect of the disease, and help improve the quality of sleep.
\end{abstract}

\section{Introduction}

Restless leg syndrome is a type of clinical multiple central neurosensory motor disorder. It has a high incidence of uremia in maintenance hemodialysis patients. Patients often have different degrees of muscle spasms, lower limbs, or feelings of ants. Such as feeling abnormal [1]. Urinary restless legs syndrome usually requires frequent movement of the legs to alleviate the relevant clinical symptoms, and the symptoms are aggravated during nighttime sleep, which may cause sleep disorders in patients, which has a great impact on their daily life and mental state. The onset of uremia restless legs syndrome may be due to the increase of microglobulin content in patients, and the accumulation of macromolecular toxins in the body. Some patients have been treated by hemodialysis, but it is difficult to effectively remove toxins. With the clinical deepening of the understanding of uremic restless leg syndrome, the focus of its treatment research turned to traditional Chinese medicine. Acupoint massage is a common intervention for traditional Chinese medicine, which can strengthen the body's immune function, and has the effects of balancing yin and yang, clearing qi and blood, and clearing meridians. In addition, because the incidence of restless legs syndrome is due to yang deficiency, the outside is not solid, the dampness is cold and suffocating, the yang is suppressed, it is difficult to reach, the blood is blocked, the limb is lost due to warming, so its The treatment should focus on soft tendons and urgency, warm through the pass, should adopt the pass through the soft tendon slow emergency soup [2]. Based on this, this study selected 78 patients with uremic restless legs syndrome in our hospital, and explored the effect of 
Tong Jing Rou Jin Ban Tang combined with acupoint massage. The report is as follows.

\section{Materials and Methods}

General Information 78 patients with uremia restless legs syndrome were enrolled in our hospital from April 2016 to January 2018. The random number table method was divided into control group $(n=39)$ and study group $(n=39)$. The control group consisted of 17 males and 22 females; aged 29-68 years, mean $(48.32 \pm 10.71)$ years old; primary disease: 1 case of lupus nephritis, 1 case of polycystic kidney disease, 4 cases of chronic interstitial nephritis, hypertension There were 9 cases of renal damage, 8 cases of diabetic nephropathy, 13 cases of nephrotic syndrome, and 3 cases of chronic glomerulonephritis. The study group included 19 males and 20 females; aged 27-65 years, mean $(48.01 \pm 11.10)$ years old; primary disease: 1 case of lupus nephritis, 2 cases of polycystic kidney, 5 cases of chronic interstitial nephritis, hypertension There were 6 cases of renal damage, 6 cases of diabetic nephropathy, 13 cases of nephrotic syndrome, and 6 cases of chronic glomerulonephritis. There was no significant difference in clinical data between age groups, genders, and primary diseases ( $\mathrm{P}>0.05)$, and the study was approved by the Ethics Committee of our hospital.

Inclusion criteria (1) Comply with the diagnosis criteria of restless leg syndrome developed by the urology of internal medicine [3] and the international restless leg syndrome research group [4]; (2) hemodialysis time $\geq 3$ Month; (3) Be aware of the study and sign the consent form.

Exclusion criteria (1) patients with severe renal and liver function disorders; (2) patients with anemia; (3) patients with systemic severe infectious diseases; (4) patients with severe malnutrition; (5) patients with Parkinson Diseases, heart failure; (6) vitamin B12 deficiency or other causes of neurological disease; (7) combined with severe autoimmune disease.

Methods Both groups were treated with hemodialysis. The device was selected from Fresenius polysulfone membrane FX60 dialysis machine, and the ultrafiltration coefficient was adjusted to 46 $\mathrm{ml} /(\mathrm{h} \bullet \mathrm{mmHg})$. The membrane surface area was $1.6 \mathrm{~m} 2$. The amount is about $12-20 \mathrm{~L}$, and the blood flow rate is set to $50-100 \mathrm{ml} / \mathrm{min}$ for $4 \mathrm{~h} /$ time. On this basis, different intervention schemes are adopted for the two groups.

Acupuncture points were taken in the control group, and the acupoints were selected from the frontal depressions of the foot, the second and third metatarsal suture heads and the first $1 / 3$ of the heel line and the second 2/3 junction of the heel. The 1st and 2nd zygomatic commissures are in front of the depression at Taichong Point; the upper part of the gastrocnemius muscle is between the abdomen; the top is the Chengshan point; the middle point of the transverse ridge is at the middle of the Venezuela; the 4 points of the medial iliac are transverse, the posterior of the humerus is the posterior of Sanyin; Yinlingquan at the lower depression; Yanglingquan at the lower part of the humerus and the lower part of the depression at the lower part of the cheekbones; 4 horizontal lines at the upper side of the zygomatic bone; and Zusanli on the outer side of the iliac bone; 1 on the lower lateral side of the humerus; Knees, upper and lower calves, muscles relaxed, with the thumb from top to bottom, press and point the above points, each acupuncture point $1 \mathrm{~min} /$ time, palm slap the calf, rub the calf back and forth, $15 \mathrm{~min} /$ time, interval $1 \mathrm{~d} 1$ time.

The study group was added to the control group based on the Rouxiujie urgent soup. The drug group prescriptions were: Spatholobus suberectus 30 g, calcined keel 30 g, chuanchuan 15 g, dog's ridge 15 g, and chuanxiong ginseng 30 g. Ume 12 g, licorice 10 g, white peony 30 g, pueraria 12 g, papaya $12 \mathrm{~g}$, barley $30 \mathrm{~g}$, Asarum $5 \mathrm{~g}$, cassia twig $12 \mathrm{~g}$, dried ginger $12 \mathrm{~g}$, tuber $10 \mathrm{~g}$, decocted in water, 1 dose / d, $300 \mathrm{ml}$ of juice, 2 times in the morning and evening. Both groups were treated for 2 months.

Observation indicators (1) After the end of the treatment period, the clinical efficacy of the two groups and the severity of the disease before and after treatment were counted. The feelings of the lower extremity ants, acid sensation, pain and numbness disappeared, and the effect on sleep was not effective; the lower extremity ants felt, Acidic sensation, pain and numbness have been alleviated, and the effect on sleep is less effective; the above criteria are invalid; total effective rate $=$ (effective + effective) $/$ total number of cases $\times 100 \%$; disease degree score based on RLS 
diagnosis The standard was evaluated. There was no discomfort within 12 days ( 0 to 4 points), no 0 points, rarely 1 point, occasionally 2 points, often 3 points, and 4 points per night; 2 feeling level ( 0 to 3 points), no feeling is 0 points, mild feeling is 1 point, moderate feeling is 2 points, severe feeling is 3 points; 3 duration ( 0 to 3 points), no or only lasts for a few seconds It is 0 points, the duration is less than 30 minutes for 1 minute, the duration is 30 to 60 minutes for 2 minutes, and the duration is longer than 60 minutes for 3 minutes [5]. (2) Quality of sleep, before treatment and after the end of treatment, according to the PSQI scale, the score range is 0 to 21 points. The lower the score, the better the quality of sleep [6].

Statistical methods Data were analyzed by SPSS18.0, measurement data $( \pm \mathrm{s})$, $\mathrm{t}$ test, count data $\mathrm{n}$ $(\%), \chi 2$ test, $\mathrm{P}<0.05$ indicates that the difference was statistically significant.

\section{Results}

Clinical efficacy The total effective rate of the study group (94.87\%) was higher than that of the control group (76.92\%, $\mathrm{P}<0.05)$. See Table 1 .

Table 1 Comparison of clinical effects between the two groups [n (\%)]

\begin{tabular}{lccccc}
\hline Gr & $\begin{array}{c}\text { Number } \\
\text { of case }\end{array}$ & $\begin{array}{c}\text { Significant } \\
\text { effect }\end{array}$ & effective & invalid & $\begin{array}{c}\text { Total } \\
\text { efficiency }\end{array}$ \\
\hline $\begin{array}{l}\text { research } \\
\text { group }\end{array}$ & 39 & $21(53.85)$ & $16(41.03)$ & $2(5.13)$ & $37(94.87)$ \\
$\begin{array}{l}\text { Control } \\
\text { group }\end{array}$ & 39 & $16(41.03)$ & $14(35.90)$ & $9(23.08)$ & $30(76.92)$ \\
$\chi^{2}$ & & & & & 5.186 \\
$P$ & & & & & 0.023 \\
\hline
\end{tabular}

RLS scores There was no significant difference in RLS scores between the two groups before treatment $(\mathrm{P}>0.05)$. After treatment, both RLS scores were lower than before treatment, and the study group was lower than the control group $(\mathrm{P}<0.05)$. See Table 2.

Table 2 Comparison of two RLS scores ( \pm s, points)

\begin{tabular}{lccccc}
\hline Gr & $\begin{array}{c}\text { Number } \\
\text { of cases }\end{array}$ & $\begin{array}{c}\text { Before } \\
\text { treatment }\end{array}$ & $\begin{array}{l}\text { After } \\
\text { treatment }\end{array}$ & $\mathrm{t}$ & $\mathrm{p}$ \\
\hline $\begin{array}{l}\text { research } \\
\text { group }\end{array}$ & 39 & $7.08 \pm 1.01$ & $3.78 \pm 0.86$ & 15.536 & 0.000 \\
$\begin{array}{l}\text { Control } \\
\text { group }\end{array}$ & 39 & $7.13 \pm 0.97$ & $5.11 \pm 1.10$ & 8.602 & 0.000 \\
$t$ & & 0.223 & 5.949 & & \\
$P$ & & 0.824 & 0.000 & & \\
\hline
\end{tabular}

PSQI scores There was no significant difference in PSQI scores between the two groups before treatment $(\mathrm{P}>0.05)$. After the end of the treatment period, the scores of PSQIs of the two groups were lower than before treatment, and the study group was lower than the control group $(\mathrm{P}<0.05)$. See Table 3.

Table 3 Comparison of PSQI scores between the two groups ( $\pm \mathrm{s}, \mathrm{min}$ )

\begin{tabular}{lccccc}
\hline Gr & $\begin{array}{c}\text { Number } \\
\text { of cases }\end{array}$ & $\begin{array}{c}\text { Before } \\
\text { treatment }\end{array}$ & $\begin{array}{l}\text { After } \\
\text { treatment }\end{array}$ & $\mathrm{t}$ & $\mathrm{p}$ \\
\hline $\begin{array}{l}\text { research } \\
\text { group }\end{array}$ & 39 & $16.89 \pm 2.25$ & $9.22 \pm 1.37$ & 18.183 & 0.000 \\
Control & 39 & $16.21 \pm 2.40$ & $12.31 \pm 1.56$ & 8.509 & 0.000 \\
group & & 1.291 & 9.295 & & \\
$t$ & & 0.201 & 0.000 & & \\
$P$ & & & & \\
\hline
\end{tabular}




\section{Discussion}

Uremic restless legs syndrome is a category of "sickness", "sickness", "blood stasis" in Chinese medicine. Chinese medicine believes that its cause lies in kidney, spleen, liver, heart dysfunction, blood deficiency, and muscle dystrophy. Nursing, combined with cold and wet evil invasion, meridian block, limb restlessness. At the same time, Chinese medicine believes that the spleen can be transported to the body muscles, and the liver and blood can moisturize the body's muscles and veins, while the kidney and liver are homologous, and the kidney and spleen are mutually nourished. Therefore, kidney and spleen, nourishing the liver and nourishing the kidney can improve the "ricky disease" and " Clinical manifestations such as blood stasis [7]. Acupoint massage is an important therapeutic measure for traditional Chinese medicine. It is guided by meridians and acupoints. It achieves the effects of treating diseases, balancing yin and yang, and dredging meridians through infiltration, potency, long-lasting, gentle and even manipulation. This study selected Yongquan (renal meridians), Taichong (liver meridians), Chengshan and Venezuela (bladder meridians), Yanglingquan (bile meridians), Sanyinjiao and Yinlingquan (Spleen meridian), Zusanli (stomach) After), combined use can play a total of dredge meridians, Ningxin Anshen, spleen and kidney, nourishing the kidney and other effects. At the same time, some studies also pointed out that uremic restless leg syndrome and metabolites produced in the lower extremity caused by lower extremity vasospasm, hypoxia-ischemia and peripheral nerve disorders are closely related, and through acupuncture can accelerate the excretion of metabolites, improve blood of the lower extremities The circulation, relaxation of muscles, and acupressure are more comfortable and are easily accepted by the majority of patients [8].

In addition, uremic hemodialysis patients suffer from kidney qi deficiency, and the kidney is the basis of yang. If the kidney loss takes a long period of time, the yang will be more deficient. Therefore, this study has adopted flexible tendons with softness, urgency and warmth. Chronic urgency soup treats patients with uremic restless legs syndrome. Through the soft and slow emergency soup aconite to go without guarding, cold and pain relief, make up the fire to help the Yang, dry ginger scattered and able to defend, help Yang Tongmai, Wenzhongzhonghan effect, the combination of the two can be warm Congenital to the day after birth, but also warm to the day to raise congenital, enhance the efficacy of Wenyang dispelling cold Tongmai; Millettia, Sichuan Achyranthes can lead the blood, phlegm Tongluo; calcined keel sedative, Ruanjian effect Chuanxiong and dog's ridges are good at strengthening kidneys and bones; Wumei can relieve pain, soothe the nerves and nourishing blood, "Shen Nong's Materia Medica" contains: ebony can eliminate irritability, relief, and limb pain; licorice and white peony; Kudzu root and papaya have the effect of relaxing the muscles and easing tendons; Qiren can improve the spleen and dampness; Asarum and cassia twig are good at warming the meridian. Modern pharmacology studies confirmed that the combination of dried ginger and aconite in Rouxiujiang Decoction can increase blood flow velocity, increase blood flow, help improve microcirculation, and produce good Wenyang Tongmai efficacy; licorice, expectorant can relieve pain and relaxation 痉孪; Calcined keel is mainly composed of calcium salt, calcium, anticonvulsant, sedative effect [9-10]. The results of this study showed that the RLS score and PSQI score of the study group decreased more significantly after the end of the treatment course, and the total effective rate of the study group was higher than that of the control group $(\mathrm{P}<0.05)$, suggesting that the acupoint massage and communication should be adopted on the basis of conventional intervention. Through the joint intervention program of Roujin brave chill and acute decoction, the degree of illness and clinical symptoms of patients with uremic restless leg syndrome can be more effectively relieved, and the therapeutic effect of the disease can be improved, and it has certain positive significance for improving the quality of sleep of patients.

In summary, the combination of acupressure massage and the treatment of urethrogenic bradykinesis in the treatment of uremic restless legs syndrome can effectively relieve the clinical symptoms of patients, reduce the degree of illness, improve the efficacy of treatment, and help improve the quality of sleep. 


\section{References}

[1] Zhou Guoli, Jiao Lizhi, Li Xinyu, et al. Clinical observation on the effect of Bushen Zhuanggu Decoction on restless leg syndrome and bone metabolism in maintenance hemodialysis patients with uremia[J]. Shanghai Journal of Traditional Chinese Medicine, 2018, 52(3) :47-50.

[2] Wang Huimin. Therapeutic effect of Wenyang Tong on uremic restless leg syndrome treated by soft tendon and emergency therapy [J]. Chinese Journal of Integrated Traditional and Western Medicine on Nephrology, 2013, 14(7): 632-633.

[3] Ye Rengao, Lu Zaiying. Internal Medicine [M]. 6th ed. Beijing: People's Medical Publishing House, 2004: 542.

[4] Walters A S, Lebrocq C, Dhar A, et al. Validation of the International Restless Legs Syndrome Study Group rating scale for restless legs syndrome [J]. Sleep Med, 2003, 4(2): 121-132.

[5] Chen Rui. Efficacy analysis of integrated traditional Chinese and Western medicine for uremic restless leg syndrome [J]. Shenzhen Journal of Integrated Traditional Chinese and Western Medicine, 2017, 27(24): 32-33.

[6] Ye vane, Yin Jinfeng, Yi Wei, et al. Clinical research on "restless leg syndrome" in improving hemodialysis patients with uremia by touching [J]. Chinese Journal of Modern Medicine, 2015, 25(36):97- 101.

[7] Ruan Zhiying, Yu Xiaomei, Zhang Wenhui, et al. Clinical efficacy of point massage in relieving hemodialysis patients with restless legs syndrome [J]. Nursing Research, 2012, 26(30):2838-2839.

[8] Wu Aichun. Therapeutic effect of footbath plus acupoint massage on restless leg syndrome in uremic patients [J].Journal of Clinical Nephrology, 2017, 17(5):308-310.

[9] Liu Jia, Wang Huimin, Wu Yiwei, et al. Therapeutic effect observation of 30 cases of restless leg syndrome treated by Wenyangtong by Rouxiujiao method[J]. New Journal of Traditional Chinese Medicine, 2013,45(5):93-94.

[10] Lü Zhichao, Wang Huimin, Liu Jia, et al. Clinical observation of 38 cases of uremic restless leg syndrome treated by Wenyangtong by Rouxiujiao method[J]. China Emergency Medicine, 2013, 22(10):1775-1776. 